\section{WEB WATCH}

Internet Mental Health

- http://mentalhealth.com/

As we advance in our fight against neurological

disorders, it becomes more apparent that we have a lot of catching up to do with regard to psychiatric conditions.

There are many reasons why the gap in our understanding of psychiatric problems is so great. For example, we lack animal models for their study. Also, the description of psychiatric disorders that we now have is far from complete, as we haven't identified the precise brain systems that are affected in many of these conditions. In fact, it isn't unusual for psychiatric disorders to be divided into 'organic' and 'functional' categories, a superseded classification that only highlights our lack of success in identifying the neural substrates of the second category. A huge challenge for neuroscientists over the coming years will be to identify the neurobiological underpinnings of psychiatric diseases, bridging at last the long-standing divide between brain research and disorders of the mind.

But where to start? To catch up, we must essentially begin at square one - by learning the basics about the plethora of psychiatric disorders that have been described. A good place to start is Internet Mental Health, a web site maintained by psychiatrist Phillip Long in Vancouver, Canada. The site hosts general information about tens of psychiatric disorders. So you can read about their features, their diagnosis and the methods that are used to treat them. The site also has a clever search engine that is linked to PubMed, which allows you to locate papers on quite specific aspects of the different disorders, aspects you might not even have thought of!

Neuroscience has a great potential to make an impact on psychiatry. The sooner we take a look into the tribulations of the mind, the sooner we'll start to fulfil this potential.

CORTICAL PLASTICITY

\title{
Right (or left) on time
}

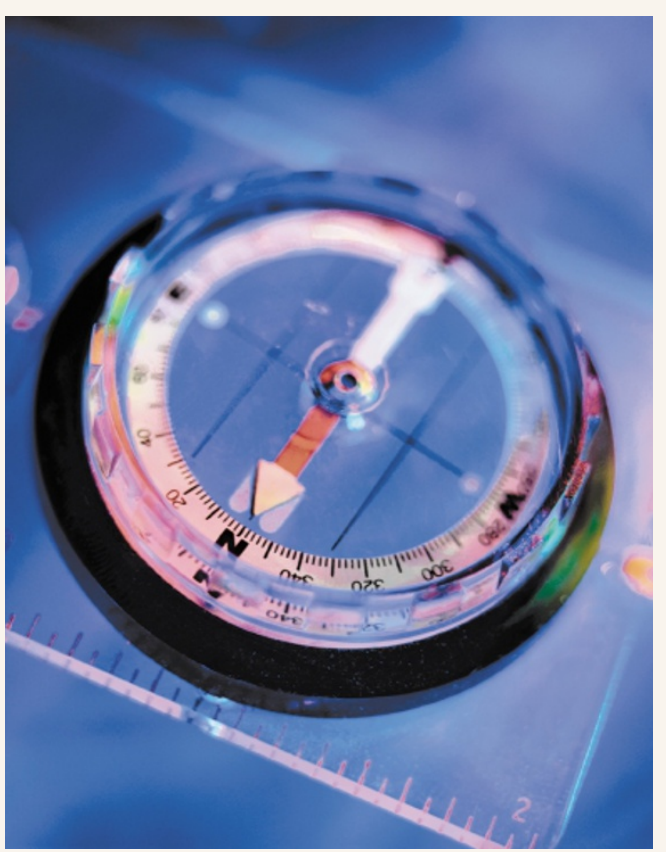

In neural plasticity, as in so many things in life, timing is everything. When presynaptic and postsynaptic stimulation of a neuron are paired, the synaptic connections will be modified - but the direction and magnitude of the change will depend crucially on the relative timing of the two stimuli. Now, two studies published in Neuron have shown that orientation plasticity in the visual cortex seems to follow similar rules.

The two studies took slightly different approaches. Yao and Dan used pairs of visual stimuli - gratings at slightly different orientations - and recorded their effects on the orientation preference of neurons in primary visual cortex. One of the gratings was presented at exactly the orientation that the neuron in question was most responsive to, and the other was rotated 15 degrees clockwise (+) or anticlockwise (-) from this orientation. Conditioning with repeated pairings of these gratings caused the orientation preferences of cortical neurons to shift, with the direction of the shift being determined by the order of the gratings.

For example, if the 'preferred orientation' grating was shown a few milliseconds after the ' +15 degrees' grating, the tuning of the neuron shifted clockwise. If the order was reversed, however, the tuning shifted anticlockwise. This effect occurred only if the two gratings were shown within 20 milliseconds of each other, which is similar to the time window for spiketiming-dependent plasticity at central synapses. Yao and Dan also used similar stimuli to condition human observers, and found that their perception of orientation also shifted, consistent with the changes in orientation sensitivity that occurred in cat cortex. In the second study, by Schuett $e$ t al., a brief visual stimulus (again, a grating at a specific orientation) was paired with electrical stimulation in the visual cortex of cats. This would result in the pairing of presynaptic stimulation, through the thalamocortical inputs, and direct postsynaptic stimulation. Using optical imaging and single-cell recordings, the authors found that such pairing also altered the orientation specificity of cells in primary visual cortex; again, the timing and order of the stimuli determined the direction of the shift. Interestingly, cells near the 'pinwheel centres' of the orientation maps - where many orientation domains come together in a small area - showed less plasticity than cells in other parts of the maps. Schuett $e t$ al. also found that cells in layer 4 of the cortex were less influenced by the pairing than cells in the upper and lower layers. They interpret this as indicating that the shift in orientation preference is caused by alterations in cortico-cortical synapses outside layer 4 , rather than thalamocortical synapses. In support of this theory, they found that the effect of the pairing could be transferred between eyes.

In a related paper, also published in Neuron, Song and Abbott describe a model in which spike-timingdependent plasticity can introduce the required element of competition into activity-dependent cortical development. Because spike-timing-dependent plasticity is asymmetrical - it can cause either potentiation or depression of synaptic strength, depending on whether the presynaptic stimulation precedes or follows the postsynaptic stimulation - it allows selective groups of neurons that fire together to drive the development of less selective neurons. Song and Abbott conclude that spike-timing-dependent plasticity can account for many features of the development of cortical maps, and can also reproduce the remapping seen following lesions of the cortical input.

Together, these papers provide an important link between the spike-timing-dependent plasticity seen in single-cell in vitro studies, and the physiological and perceptual plasticity that occurs in the visual system, both in adulthood and during development. Further studies could strengthen this link, and help us to understand plasticity at all levels.

Rachel Jones

(2) References and links

ORIGINAL RESEARCH PAPER Yao, H. \& Dan, Y. Stimulus timingdependent plasticity in cortical processing of orientation. Neuron 32 315-323 (2001) | Schuett, S. et al. Pairing-induced changes of orientation maps in cat visual cortex. Neuron 32, 325-337 (2001) | Song, S. \& Abbott, L. F. Cortical development and remapping through spike timingdependent plasticity. Neuron 32, 339-350 (2001) FURTHER READING Ferster, D \& Miller, K. D. Neural mechanisms of orientation selectivity in the visual cortex. Annu. Rev. Neurosci. 23, orientation selectivity in the visual cortex. Annu. Rev. Neurosci. 23 ,
$441-447$ (2000) 